\section{International Scientific Journal Theoretical \& Applied Science}

p-ISSN: 2308-4944 (print) $\quad$ e-ISSN: 2409-0085 (online)

Year: $2015 \quad$ Issue: 12 Volume: 32

Published: $30.12 .2015 \quad \underline{\text { http://T-Science.org }}$

SECTION 7. Mechanics and machine construction.
Nikolai Andreevich Gorin

Candidate of technical sciences, Engineer JSC «MPO-Rumyantsev», Moscow, Russia gorin.nikolai@mail.ru

Pavel Anatolievich Melnikov Candidate of technical sciences, Director of the Institute of Chemistry and Environmental Engineering Togliatti State University, Russia topavel@mail.ru

Aleksey Aleksandrovich Lukyanov Lead Engineer

Togliatti State University, Russia a.lukyanov@tehnomasch.ru

Aleksey Sergeevich Sevostyanov Undergraduate student

Togliatti State University, Russia sevalexey@yandex.ru

Olesya Olegovna Levitskih Engineer

Togliatti State University, Russia 1oo-05@mail.ru

\title{
THE PROBLEM OF WIDE BURNISHING MODELING OF THERMAL FIELDS
}

\footnotetext{
Abstract: In this paper modern problems of wide burnishing modeling is presented. The comparison of the basic models used to calculate the temperature is performed. The main directions of further development of research are determined. The study was supported by RFBR under the research project Op 15-38-50657 15 "mol $n r "$.

$\bar{K}$ ey words: finite element method, thermal field, surface plastic deformation, wide burnishing.

Language: Russian

Citation: Gorin NA, Melnikov PA, Lukyanov AA, Sevostyanov AS, Levitskih OO (2015) THE PROBLEM OF WIDE BURNISHING MODELING OF THERMAL FIELDS. ISJ Theoretical \& Applied Science 12 (32): 6770 .

Soi: http://s-o-i.org/1.1/TAS-12-32-13 Doi: crossef http://dx.doi.org/10.15863/TAS.2015.12.32.13
}

\section{ЗАДАЧИ МОДЕЛИРОВАНИЯ ТЕПЛОВЫХ ПОЛЕЙ ПРИ ШИРОКОМ ВЫГЛАЖИВАНИИ}

Аннотация: В работе рассмотрены современые задачи в области моделирования метода обрабработки широким выглаживанием. Выполнено сравнение основных моделей, используемьх для рассчета температур. Определены направления дальнейшего развития исследований. Исследование выполнено при финансовой поддержке РФФИ в рамках научного проекта № Ор 15-38-5065入15 «мол_нр».

Ключевые слова: метод конечных элементов, тепловое поле, поверхностно-пластическое деформирование, иирокое выглаживание.

Технологии обработки ППД получили существенное развитие в 1950-1980ых годах. Технологическая простота метода ППД дает возможность применения его на всех машиностроительных предприятиях, в том числе в ремонтных цехах на универсальных станках [1, $2,3]$.

В начале 2000-ных годов был разработан новый вид ППД - широкое выглаживание.
Главное отличие от известных технологий обработки - отсутствие продольной подачи и высокая скорость обработки. Однако, для осуществления данного типа обработки требуется в десятки раз большие усилия, чем при обработке другими методами ППД, что соответственно требует большего внимания к вопросам теплообразования $[4,5]$. 


\begin{tabular}{l|lrl|l|ll} 
& ISRA (India) & $=\mathbf{1 . 3 4 4}$ & SIS (USA) & $=\mathbf{0 . 9 1 2}$ & ICV (Poland) & $=\mathbf{6 . 6 3 0}$ \\
Impact Factor: & ISI (Dubai, UAE) $=\mathbf{0 . 8 2 9}$ & PUHU (Russia) $=\mathbf{0 . 1 7 9}$ & PIF (India) & $=\mathbf{1 . 9 4 0}$ \\
& GIF (Australia) & $\mathbf{0 . 5 6 4}$ & ESJI (KZ) & $=\mathbf{1 . 0 4 2}$ & & \\
& JIF & $=\mathbf{1 . 5 0 0}$ & SJIF (Morocco) $=\mathbf{2 . 0 3 1}$ & &
\end{tabular}

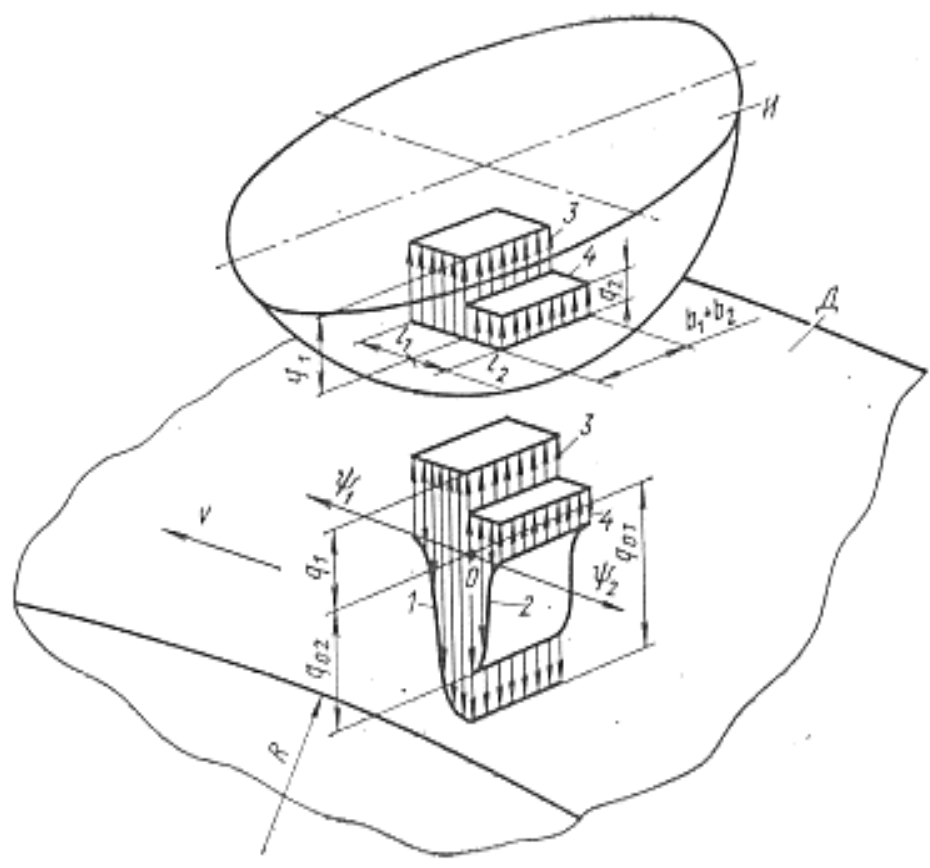

Рисунок 1 - Схематизация процесса выглаживания.

Понимание условий теплообразования и распределения тепла, и их количественная оценка на финишных операциях обработки деталей критично для минимизации негативного и необратимого влияния тепла на формирование эксплуатационных свойств, что актуально при механической обработке, в том числе, при широком выглаживании [6, 7] В справочной литературе отсутствуют аналитические зависимости для широкого выглаживания, позволяющие рассчитать температуру в любой точке области обработки инструмента и заготовки [8].

Как отмечено в работе Резникова А.Н. [9] «для рациональной организации процесса выглаживания, обеспечивающей наибольшую производительность и создание условий для нормальной работы кристалла алмаза, необходимо определить температуры, возникающие на контактных поверхностях последнего».

Разработанный Мельниковым П.А. метод анализа тепловых полей при широком выглаживании позволяет рассчитать температуру в любой точке области и базируется на методе источников [10]. Резников А.Н. и Барац Я.И. в своих публикациях рассматривают только методы моделирования полей при выглаживании сферическим инструментом с подачей, используя схематизацию формы инструмента и площадки контакта, предложенную Д.Д. Папшевым [11]. При схематизации процесса выглаживания и определении формы и конфигурации источников тепла источник условно делят на две части (рис.1).
Учитывая различие в технологиях обработки и возникла необходимость разработки модели теплообразования при широком выглаживании [12]. Краткое описание имеющийся модели:

- отдельные компоненты модели инструмент и деталь;

- учет накопления температур в детали при проведении нескольких циклов обработки с помощью расчета стационарной тепловой картины;

- возможность учета обработки с применением и без применения смазывающеохлаждающих технических средств с помощью стоков теплоты;

- моделирование движущегося и быстродвижущегося с помощью критерия Пекле $(\mathrm{Pe} \geq 10)$ источника тепла, учета Ре для различных сочетаний условий обработки (скорость процесса обработки и длина)

- учет доли теплоты, уходящей в инструмент решения балансовой задачи и принципа отражения источников;

- учет теплофизических характеристик материалов, применяемой технологической схемы обработки и их способности упрочнения с помощью показателя деформационного упрочнения имеющего экстремальную зависимость от температуры;

- различное расположение и количество инструментов (рис. 2), необходимо учесть, что при широком выглаживании каждая точка поверхности подвергается нескольким циклам нагружения. 


\begin{tabular}{l|lrl|l|ll} 
& ISRA (India) & $=\mathbf{1 . 3 4 4}$ & SIS (USA) & $=\mathbf{0 . 9 1 2}$ & ICV (Poland) & $=\mathbf{6 . 6 3 0}$ \\
Impact Factor: & ISI (Dubai, UAE) $=\mathbf{0 . 8 2 9}$ & PUHU (Russia) $=\mathbf{0 . 1 7 9}$ & PIF (India) & $=\mathbf{1 . 9 4 0}$ \\
& GIF (Australia) & $\mathbf{0 . 5 6 4}$ & ESJI (KZ) & $=\mathbf{1 . 0 4 2}$ & & \\
& JIF & $=\mathbf{1 . 5 0 0}$ & SJIF (Morocco) $=\mathbf{2 . 0 3 1}$ & &
\end{tabular}

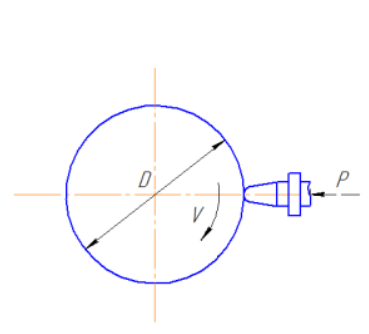

Схема $A$

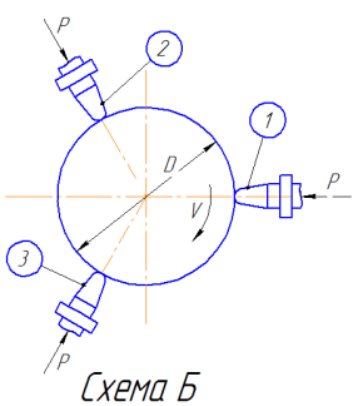

Схема 5

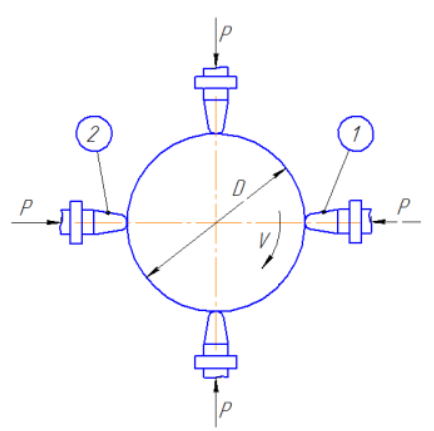

Схема $B$

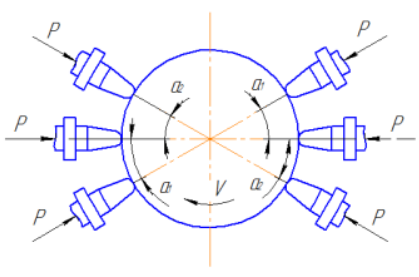

¿хема Г

Рисунок 2 - Основные технологические схемы процесса широкого выглаживания учтенные в модели.

По мнению авторов, данная модель не позволяет адекватно выполнить расчет, т.к. представление источника тепла в виде стационарного некорректно и может привести к большой погрешности в расчетах, необходимо разработать модель учитывающую изменение площади контакта и усилия при постоянных значениях затрачиваемой работы.

Информационный поиск в базах данных отечественных и зарубежных источниках показал, что хотя и разработки в области тепловых моделей для поверхностнопластического деформирования продолжаются, они направлены на исследование процессов при комбинированной обработке (например, ППД и ультразвуковое упрочнение) либо являются переосмыслением классических работ, однако в подавляющем большинстве отечественных работ используется метод источников. В зарубежной литературе моделирование процессов ППД для оценки возникающих в процессе явлений, таких как остаточные напряжения, выполняют с помощью метода конечных элементов (МКЭ), а метод источников практически не применяется. Научные школы РФ, выполняющие исследования в области ППД так же применяют МКЭ [13], но нет сведений, подтверждающих применения МКЭ для исследования теплоты при ППД.

Несмотря на то, что метод источников был разработан в Тольяттинском государственном университете (ТГУ) Резниковым А.Н. авторам представляется перспективным осуществить переход от аналитической модели к численной и видоизменить текущую модель обработки широким выглаживанием, реализовав ее с помощью МКЭ.

Таким образом, основные модели расчета температур при выглаживании, упомянутые выше позволяют рассчитать среднюю, максимальную или другие отдельные характерные значения температур на площадке контакта, и не дают полной картины теплового поля в инструменте или заготовке, не возможно моделирование с помощь данных моделей широкого и многоинструментального выглаживания - требуется разработка новой модели теплообразования при широком выглаживании, соответствующей требованиям, упомянутым в данной статье.

Исследование выполнено при финансовой поддержке РФФИ в рамках научного проекта № Ор 15-38-5065入15 «мол_нр». 


\begin{tabular}{l|lrl|l|ll} 
& ISRA (India) & $=\mathbf{1 . 3 4 4}$ & SIS (USA) & $=\mathbf{0 . 9 1 2}$ & ICV (Poland) & $=\mathbf{6 . 6 3 0}$ \\
Impact Factor: & ISI (Dubai, UAE) $=\mathbf{0 . 8 2 9}$ & PUHIL (Russia) $=\mathbf{0 . 1 7 9}$ & PIF (India) & $=\mathbf{1 . 9 4 0}$ \\
& GIF (Australia) & $\mathbf{0 . 5 6 4}$ & ESJI (KZ) & $=\mathbf{1 . 0 4 2}$ & & \\
\hline JIF & $=\mathbf{1 . 5 0 0}$ & SJIF (Morocco) $=\mathbf{2 . 0 3 1}$ & & \\
\hline
\end{tabular}

\section{References:}

1. Bobrovskij NM, Mel'nikov PA (2004) Stojkost' tverdosplavnogo vyglazhivajushhego instrumenta pri rabote bez SOZh. Avtomobil'naja promyshlennost'. No 8. pp. 3335.

2. Bobrovskij NM, Mel'nikov PA, Bobrovskij IN, Ezhelev AV, Lukyanov AA (2011) Issledovanie vlijanija rezhimov obrabotki na sherohovatost' poverhnosti zakalennyh valov $\mathrm{v}$ uslovijah massovogo proizvodstva. Sovremennye problemy nauki i obrazovanija. No 5 . Available: www.science-education.ru/99-4791. (Accessed: 22.12.2015).

3. Brzhozovskij BM, Martynov VV, Zaharov OV (2014) Upravlenie tochnost'ju nastrojki razmera na tokarnyh stankah na osnove optimal'noj shemy izmerenija. STIN. No 2. pp. 14-16.

4. Bobrovskij NM, Mel'nikov PA (2010) Prognozirovanie processa iznashivanija rabochej poverhnosti instrumenta pri vyglazhivanii bez smazochno-ohlazhdajushhih sredstv. Vektor nauki TGU. 2010. No 2(12). pp. 43-48.

5. Sevost'janov AS, Lukyanov AA, Bobrovskij IN (2014) Sovremennoe sostojanie issledovanij v oblasti nanesenija mikrorel'efa v otechestvennoj nauke. Theoretical \& Applied Science. 2014. No 10(18). $\quad$ pp. http://elibrary.ru/download/92075784.pdf

6. Kuznecov VP, Makarov AV, Pozdeeva NA, Savraj RA, Jurovskih AS, Malygina IJ, Kirjakov AE (2011) Povyshenie prochnosti, teplostojkosti i iznosostojkosti detalej iz cementovannoj stali 20X nanostrukturirujushhim frikcionnym vyglazhivaniem na tokarno/frezernyh centrah.
Uprochnjajushhie tehnologii i pokrytija. No 9. pp. 3-13.

7. Kuznecov VP (2009) Metodika opredelenija parametrov instrumenta dlja uprugogo vyglazhivanija detalej na osnove modelirovanija nelinejnoj dinamiki processa. Mashinostroenie i inzhenernoe obrazovanie. No 3. pp. 18-26.

8. Grigor'ev SN, Bobrovskij IN (2011) Kontaktnoe vzaimodejstvie pri vyglazhivanii shirokim samoustanavlivajushhimsja instrumentom. Vestnik MGTU Stankin. No 1. pp. 84-87.

9. Reznikov AN (1981) Teplofizika processov mehanicheskoj obrabotki materialov. Moscow: Mashinostroenie. $279 \mathrm{p}$.

10. Bobrovskij NM, Mel'nikov PA (2010) Prognozirovanie processa iznashivanija rabochej poverhnosti instrumenta pri vyglazhivanii bez smazochno-ohlazhdajushhih sredstv. Vektor nauki TGU. No 2(12). pp. 4348.

11. Barac JI (1982) Finishnaja obrabotka metallov davleniem (Teplofizika i kachestvo). Saratov: Izdatel'stvo Saratovskogo universiteta. $181 \mathrm{p}$.

12. Zaharov OV (2013) Vybor optimal'noj shemy kontrolja razmerov detalej na stankah tokarnoj gruppy. Kontrol'. Diagnostika. No 11. pp. 4244.

13. Kuznecov VP, Smolin IJ, Dmitriev AI, Konovalov DA, Makarov AV, Kirjakov AE, Jurovskih AS (2011) Konechno-jelementnoe modelirovanie nanostrukturirujushhego vyglazhivanija. Fizicheskaja mezomehanika. $\begin{array}{llll}\text { No } & 6 . & \text { pp. } & \text { 87-97. }\end{array}$ http://elibrary.ru/download/62620065.pdf 